DAMTP-1999-165

hep-th/9911154

\title{
PhreMology: calibrating M-branes
}

\author{
Paul K. Townsend \\ DAMTP, University of Cambridge, \\ Silver Street, Cambridge CB3 9EW, UK
}

\begin{abstract}
The relevance of calibrations, and 'generalized' calibrations, to supersymmetric M-brane configurations, and their associated field theories, is reviewed, with emphasis on applications to domain walls and domain wall junctions of $\mathrm{D}=4 \mathrm{~N}=1$ supersymmetric field theories.
\end{abstract}

\footnotetext{
${ }^{1}$ To appear in the proceedings of 'Strings 99 '.
} 
Minimal surfaces have long been an active area of mathematics. With the advent of the 'brane revolution' they have become important to theoretical particle physics via M-theory. An important development on the mathematical side was the introduction in 1982 of the concept of a calibration [1]. In the simplest cases this deals with $p$-dimensional submanifolds of $\mathbb{E}^{n}$. A p-form $\phi$ on $\mathbb{E}^{n}$ is a calibration if, for all tangent p-planes $\xi$,

(i) $\phi_{\xi} \leq \operatorname{vol}_{\xi}$

(ii) $d \phi=0$

The contact set of the calibration is the set of all tangent p-planes for which the calibration inequality (i) is saturated, and a calibrated p-surface is one for which all tangent p-planes belong to the contact set of a calibration?. A theorem of Harvey and Lawson states that a calibrated p-surface has minimum p-volume among all p-surfaces with the same homology [1]. That is, it is a minimal p-surface.

The proof of this theorem is elementary. Consider an open set $U$ of a surface satisfying the premise. Since the calibration inequality (i) is saturated, by hypothesis, we have

$$
\operatorname{vol}(U)=\int_{U} \phi
$$

Now consider any other p-surface that coincides with the original one on the boundary $\partial U$ of $U$, and let $U^{\prime}$ be an open set of this new surface with $\partial U^{\prime}=\partial U$. Since we only consider surfaces within the same homology class there exists a $(p+1)$-surface $D$ such that $\partial D=U-U^{\prime}$. It follows that

$$
\operatorname{vol}(U)=\int_{U^{\prime}} \phi+\int_{D} d \phi
$$

By property (i) the first term on the right hand side cannot exceed $\operatorname{vol}\left(U^{\prime}\right)$, while the second term vanishes by property (ii). We therefore deduce the inequality

$$
\operatorname{vol}(U) \leq \operatorname{vol}\left(U^{\prime}\right)
$$

The theorem then follows from the fact that this is true for any choice of $U$.

Applications of this theorem to branes, pioneered in [2, 3, , $1,5,6$, rely on the fact that, in many cases of interest, the energy of a static p-brane is proportional to the p-volume of its worldspace $w$, the constant of proportionality being the p-volume tension $T$. There are, however, many cases in which the energy is not simply the p-volume. Obvious examples are D-branes and the M5-brane which have gauge-fields on their worldvolume. The theory of calibrations is still applicable in these cases if (when consistency allows it) one restricts attention to configurations for which

\footnotetext{
${ }^{2}$ Each p-plane tangent to a surface in $\mathbb{E}^{n}$ corresponds to a point on the Grassmannian of p-planes through the origin of $\mathbb{E}^{n}$, so the contact set of a calibration is a subset of this Grassmannian. This relies on the fact that $\mathbb{E}^{n}$ has trivial holonomy, but a modified theory exists for spaces of reduced holonomy.

${ }^{3}$ No attempt will be made here to survey the many subsequent applications, except those of direct relevance to $\mathrm{D}=4 \mathrm{~N}=1$ domain walls and their intersections to be discussed below.
} 
worldvolume gauge fields vanish. Discounting worldvolume gauge fields, the bosonic p-brane action takes the universal form

$$
S=-T \int_{W}[\operatorname{vol}(g)+A]
$$

where $\operatorname{vol}(g)$ is the $(\mathrm{p}+1)$-volume form in the metric $g$ induced on the worldvolume $W$ swept out by the worldspace $w$ in the course of its time evolution, and $A$ is a $(\mathrm{p}+1)$ form induced on $W$ from a background $(\mathrm{p}+1)$-form. For stationary backgrounds one has a timelike Killing vector field $k$ for which $\mathcal{L}_{k} F$ vanishes, where $F=d A$ is the $(\mathrm{p}+2)$-form field strength for $A$. This Kiling vector field generates a symmetry of the p-brane action for which there is a corresponding Noether energy. For static branest in static backgrounds the energy density is

$$
\mathcal{H}=T\left[\sqrt{-k^{2}} \sqrt{\operatorname{det} m}+\Phi\right]
$$

where $m$ is the induced worldspace metric, and $\Phi$ is the worldspace dual of $i_{k} A$ (in a gauge for which $\mathcal{L}_{k} A=0$ ). In other words, $\Phi$ is an 'electrostatic' energy density associated with the background gauge potential $A$. If $\Phi$ vanishes then a minimal energy p-brane is a minimal p-surface (in an appropriately rescaled metric unless $k^{2} \equiv-1$ ). If $\Phi$ does not vanish then a brane of minimal energy is not a minimal surface. However, in this case one can invoke a theory of 'generalized calibrations' [8, 9] (not to be confused with generalizations involving non-vanishing worldvolume gauge fields [10, 11]).

A 'generalized calibration' is a p-form satisfying, for all tangent p-planes $\xi$,

(i)' $\phi_{\xi} \leq \sqrt{-k^{2}}$ vol $_{\xi}$

(ii)' $d \phi=i_{k} F$

For simplicity we shall assume here that $k^{2} \equiv-1$, and refer to [9] for the general case. In this case, property (i)' reduces to property (i) and the same arguments as before lead to

$$
\operatorname{vol}(U) \leq \operatorname{vol}\left(U^{\prime}\right)+\int_{D} d \phi .
$$

In the gauge for which $\mathcal{L}_{k} A=0$ we have $i_{k} F=-d\left(i_{k} A\right)$ and hence, from property (ii)', $d \phi=-d\left(i_{k} A\right)$. Thus,

$$
\int_{D} d \phi=-\int_{U} i_{k} A+\int_{U^{\prime}} i_{k} A
$$

and we deduce the generalized bound

$$
\operatorname{vol}(U)+\int_{U} i_{k} A \leq \operatorname{vol}\left(U^{\prime}\right)+\int_{U^{\prime}} i_{k} A
$$

This is equivalent to

$$
E(U) \leq E\left(U^{\prime}\right)
$$

\footnotetext{
${ }^{4}$ See [7] for a discussion of calibrations in relation to non-static branes.
} 
where $E$ is the integral of the energy density $\mathcal{H}$ of (5), with $k^{2} \equiv-1$. This illustrates the general result of [9] that the contact set of a generalized calibration is a minimal energy p-surface.

Generalized calibrations are needed to study supersymmetric branes in those supergravity backgrounds for which the 'electrostatic' energy density $\Phi$ is non-vanishing. In this contribution I will limit myself to supergravity backgrounds for which $\Phi$ vanishes, and for which the background metric is flat, so that $k^{2} \equiv-1$. In such cases only the standard calibrations are needed. I will begin by explaining how, in these simple circumstances, the calibration bound (i) follows from the p-brane supersymmetry algebra; I refer the reader to [9] for the general case. A super p-brane in a vacuum background is invariant under supertranslations of superspace. This invariance implies the existence of spinorial Noether charges $Q$, in addition to the energy and momentum. As this symmetry is a rigid one, the charges in any region $U$ of the brane are well-defined. When account is taken of the p-form central charge in the spacetime supertranslation algebra [12], one finds that

$$
\{Q, Q\}=\int_{U}\left[\operatorname{vol} \pm \Gamma_{0} \Gamma_{I_{1} \ldots I_{p}} d X^{I_{1}} \wedge \ldots \wedge d X^{I_{p}}\right]
$$

where vol is the volume p-form in the induced worldspace metric $m$, and $X^{I}$ are the n-space coordinates. The sign depends on the orientation of the p-brane in $\mathbb{E}^{n}$. The values of $p$ and $n$ are restricted by supersymmetry, but these restrictions are those required anyway for applications to M-theory. The values of $p$ and $n$ are further restricted if we assume that the supercharges $Q$ are real. This assumption is not essential but will be made here to simplify the presentation. The matrices $\left(\Gamma_{0}, \Gamma_{I}\right)$ are the Dirac matrices of $(1+\mathrm{n})$-dimensional Minkowski spacetime.

We now introduce a real, commuting, covariantly constant spinor $\epsilon$, to be called a 'Killing spinor', normalized so that

$$
\epsilon^{T} \epsilon=1
$$

The number of such spinors will always equal the number of supersymmetry charges Q. Given such a spinor, (10) implies that

$$
(Q \epsilon)^{2}=\int_{U}[v o l \pm \phi]
$$

where

$$
\phi=\frac{1}{p !}\left(\epsilon^{T} \Gamma_{0} \Gamma_{I_{1} \cdots I_{p}} \epsilon\right) d X^{I_{1}} \wedge \cdots \wedge d X^{I_{p}} .
$$

The left hand side of (12) is manifestly positive, and since this is so for any region $U$ we must have

$$
\phi_{\xi} \leq \operatorname{vol}_{\xi}
$$

for all $\xi$. It is also obvious, since $\epsilon$ is covariantly constant, that $d \phi=0$. We conclude that $\phi$ is a p-form calibration. As we shall now see, the calibration inequality (14) is 
saturated by configurations that preserve some fraction of the spacetime supersymmetry.

The calibration just found from considerations of supersymmetry is such that

$$
\phi_{\xi}=\operatorname{vol}_{\xi}\left(\epsilon^{T} \Gamma_{\xi} \epsilon\right)
$$

where $\Gamma_{\xi}$ is the matrix

$$
\Gamma=\frac{1}{p ! \sqrt{\operatorname{det} m}} \varepsilon^{i_{1} \ldots i_{p}}\left(\partial_{i_{1}} X^{I_{1}} \ldots \partial_{i_{p}} X^{I_{p}}\right) \Gamma_{0} \Gamma_{I_{1} \ldots I_{p}}
$$

evaluated at the point to which the p-plane $\xi$ is tangent. Given the restrictions on $p$ and $n$ mentioned previously, it can be shown that

$$
\Gamma^{2}=1
$$

The eigenvalues of $\Gamma$ are therefore \pm 1 , and the bound (14) is an immediate consequence of this. As we have already derived this bound, the more relevant point here is that the condition for its saturation is

$$
\Gamma \epsilon=\epsilon
$$

This is the key equation for what follows. For many simple applications we may choose coordinates for which the Killing spinor $\epsilon$ is constant. For simplicity, let us suppose that such coordinates have been chosen and that $\epsilon$ is constant. For a given tangent p-plane $\xi$, equation (18) becomes $\Gamma_{\xi} \epsilon=\epsilon$, which states that $\epsilon$ must belong to the +1 eigenspace of $\Gamma_{\xi}$; let us call this $S_{\xi}^{+}$. This space has a dimension equal to half the number of supersymmetry charges so, locally, the brane preserves $1 / 2$ supersymmetry. This will also be true globally if the brane geometry is planar, but in general $\xi$ will depend on position on the brane and hence $S_{\xi}^{+}$will vary with position. In this case the space of solutions of $(\sqrt{18})$ is the intersection of the spaces $S_{\xi}^{+}$for all $\xi$. For a generic p-brane this space is the empty set but for special cases there will be a non-empty intersection. Non-planar branes (or intersections of planar branes) for which (18) has at least one solution are calibrated by $\phi$, but will generally preserve less than $1 / 2$ supersymmetry. As we go from one point on the p-surface to another we go from one p-plane $\xi$ to another p-plane $\xi^{\prime}$. Correspondingly,

$$
\Gamma_{\xi} \rightarrow \Gamma_{\xi^{\prime}}=R^{-1} \Gamma_{\xi} R
$$

where $R$ is some $S O(n)$ rotation matrix in the spinor representation. The p-surface will be a calibrated one only if there exist non-zero solutions $\epsilon$ to $R \epsilon=\epsilon$, because only in this case will $S_{\xi}^{+}$and $S_{\xi^{\prime}}^{+}$have a non-empty intersection.

In the context of M-theory, $\epsilon$ is a 32 component Majorana spinor of $S O(1,10)$, which is real in a real representation of the Dirac matrices. For static solutions we

\footnotetext{
${ }^{5}$ The Lagrangian version of this equation was originally derived in [13, 14] from considerations of 'kappa-symmetry'. The derivation here follows [9].
} 
may consider $\epsilon$ to be a spinor of $S O(10)$, so $n \leq 10$. Preservation of some nonzero fraction $\nu$ of supersymmetry by a static M-brane configuration requires $R$ to take values in a subgroup $G$ of $S O(n) \subset S O(10)$ such that the decomposition of the spinor representations of $S O(n)$ contained in the spinor of $S O(10)$ includes at least one singlet. If the total number of singlets is $32 \nu$ then the configuration will preserve the fraction $\nu$ of supersymmetry; it is ' $\nu$-supersymmetric'. The subgroups of $S O(10)$ with the required property are

$$
\begin{aligned}
S U(5) & \subset S O(10) \\
\operatorname{Spin}(7) & \subset S O(8) \\
S U(4) & \subset S O(8) \\
G_{2} & \subset S O(7) \\
S U(3) & \subset S O(6) \\
S U(2) & \subset S O(4)
\end{aligned}
$$

For each supersymmetric, and hence calibrated, p-surface the tangent planes must parameterize a coset space $G / H$, where $G$ is the rotation group discussed above and $H$ is some stability subgroup. The groups $G$ and $H$, together with the dimension $p$ of the calibrated surface, provide a classification [1, 5, 6] of the calibrations relevant to supersymmetric configurations of M5-branes. These are shown in Table 1.

Table 1: Calibrations and supersymmetry

\begin{tabular}{|c|c|c|c|c|c|}
\hline $\mathrm{p}$ & $\mathrm{n}$ & $G$ & $H$ & Calibration Type & Susy fraction \\
\hline 2 & 4 & $S U(2)$ & $U(1)$ & Kähler & $1 / 4$ \\
\hline 2 & 6 & $S U(3)$ & $S[U(2) \times U(1)]$ & Kähler & $1 / 8$ \\
\hline 4 & 6 & $S U(3)$ & $S[U(2) \times U(1)]$ & Kähler & $1 / 8$ \\
\hline 3 & 3 & $S U(3)$ & $S O(3)$ & Special Lagrangian & $1 / 8$ \\
\hline 3 & 7 & $G_{2}$ & $S O(4)$ & Associative & $1 / 16$ \\
\hline 4 & 7 & $G_{2}$ & $S O(4)$ & Co-associative & $1 / 16$ \\
\hline 4 & 8 & $S U(4)$ & $S[U(2) \times U(2)]$ & Kähler & $1 / 16$ \\
\hline 4 & 8 & $S U(4)$ & $S O(4)$ & Special Lagrangian & $1 / 16$ \\
\hline 4 & 8 & $S p i n(7)$ & {$[S U(2) \times S U(2) \times S U(2)] / \mathbb{Z}_{2}$} & Cayley & $1 / 32$ \\
\hline 5 & 10 & $S U(5)$ & $S O(5)$ & Special Lagrangian & $1 / 32$ \\
\hline
\end{tabular}

The simplest realization of these possibilities is via orthogonally intersecting M5branes. Here I will follow the approach of [5]. Consider, for example, two M5-branes intersecting according to the array

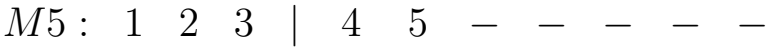

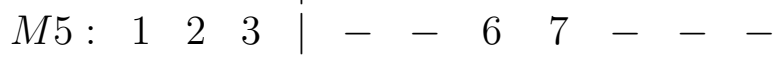

${ }^{6}$ There are additional cases when one includes M2-branes [4, 9]. See [15] for a comprehensive review. 
Each M5-brane determines a tangent p-plane $\xi$, and hence an associated matrix $\Gamma_{\xi}$ with +1 eigenspace $S_{\xi}^{+}$. The intersection of these spaces can be shown, by standard methods, to be 8 -dimensional, so the configuration preserves $1 / 4$ supersymmetry and must be a calibrated configuration. The 1-2-3 directions, separated from the others in the above array by the vertical line, are common to both M5-brane worldvolumes. They play an inessential role and may be ignored, as may the transverse 8-9- $\downarrow$ directions (following [16], we use ' $\downarrow$ ' as a convenient single character for 'ten'), so we effectively have two intersecting 2 -branes in $\mathbb{E}^{4}$. The 2 -brane in the 6 - 7 transverse directions may be considered as a 'solitonic' deformation of a test brane in the 4-5 directions. From this perspective, the orthogonal intersection of the two 2-branes is a singular limit of a configuration of a single 2-brane, which can be considered as an elliptic curve in $\mathbb{C}^{2}$, asymptotic to the two orthogonal 2-planes in the 4-5 and 6-7 directions. Either plane can be obtained from the other by a discrete $S U(2)$ rotation in $\mathbb{C}^{2}$. Such rotations preserve $1 / 4$ supersymmetry [17]. We can desingularize the intersection, while maintaining $1 / 4$ supersymmetry by allowing a continuous $S U(2)$ rotation from one asymptotic 2-plane to another. We then have a non-singular elliptic curve which, because it preserves $1 / 4$ supersymmetry, must be a calibrated 2 -surface. It must be calibrated by an $S U(2)$ Kähler calibration as this is the only case with the required properties, the Kähler and Special Lagrangian calibrations being equivalent for $S U(2)$.

This conclusion can be confirmed directly from the constraints imposed on Killing spinors by two orthogonally intersecting M5-branes [5]. Let us consider here the simpler case of two orthogonally intersecting M2-branes because the three common directions of the two M5-branes are irrelevant to the final result. We take the M2branes to intersect according to the array

$$
\begin{array}{lllllllllll}
M 2: & 1 & 2 & - & - & - & - & - & - & - & - \\
M 2: & - & - & 3 & 4 & - & - & - & - & - & -
\end{array}
$$

The calibration form $(13)$ in this case is the 2 -form

$$
\phi=\frac{1}{2} \sum_{i, j=1}^{4}\left(\epsilon^{T} \Gamma_{0 i j} \epsilon\right) d x^{i} \wedge d x^{j} .
$$

The constraints imposed on the Killing spinors by the two M2-branes are

$$
\Gamma_{012} \epsilon=\epsilon, \quad \Gamma_{034} \epsilon=\epsilon .
$$

These imply

$$
\Gamma_{12} \epsilon=\Gamma_{34} \epsilon, \quad \Gamma_{13} \epsilon=-\Gamma_{24} \epsilon, \quad \Gamma_{14} \epsilon=\Gamma_{25} \epsilon
$$

and also

$$
\epsilon^{T} \Gamma_{013} \epsilon=0 \quad \epsilon^{T} \Gamma_{014} \epsilon=0 .
$$

Given the normalization (11) of $\epsilon$, we then find that

$$
\phi=d X^{1} \wedge d X^{2}+d X^{3} \wedge d X^{4} .
$$


Introducing the complex coordinates $z=X^{1}+i X^{2}$ and $w=X^{1}+i X^{2}$ we see that $\phi$ is a closed hermitian 2-form on $\mathbb{E}^{4}$; that is, a Kähler 2-form. It is also $S U(2)$-invariant with $(d z, d w)$ transforming as a complex doublet. The 2 -form $\phi$ is therefore an $S U(2)$ Kähler calibration.

The same conclusion can be reached for M5-branes via a similar analysis of the constraints imposed on Killing spinors (after factoring out from the 5-form of (13) the volume 3-form of the three common directions). But these constraints apply to a much wider class of configurations than orthogonally intersecting M5-branes. In particular, they apply to any $1 / 4$ supersymmetric configuration of a single M5-brane that is asymptotic to the configuration of orthogonally intersecting M5-branes. The asymptotic planes may also be rotated relative to each other (see [6] for a discussion of calibrations in this context). One may consider the array as a shorthand for the entire collection of such configurations. Pushing this interpretation to the extreme, one may regard the array as nothing more than a tabular representation of the constraints (22), so that it may be considered to represent any configuration yielding these constraints regardless of the asymptotic behaviour. If an interpretation this liberal is adopted then the geometry of the array may suggest results that are spurious in particular contexts, but one may still hope to capture general features common to all cases. We shall return to this point below when discussing the associative and Cayley calibrations, but we first need to consider a simpler case with 1/8 supersymmetry.

Consider three M5-branes intersecting according to the array

$$
\begin{array}{llll|lllllll}
M 5: & 1 & 2 & 3 & 4 & 5 & - & - & - & - & - \\
M 5: & 1 & 2 & 3 & - & - & 6 & 7 & - & - & - \\
M 5: & 1 & 2 & 3 & - & - & - & - & 8 & 9 & -
\end{array}
$$

Each M5-brane determines a tangent p-plane $\xi$, and hence an associated matrix $\Gamma_{\xi}$ with +1 eigenspace $S_{\xi}^{+}$. The intersection of these spaces can be shown, by standard methods, to be 4 -dimensional, so the configuration preserves $1 / 8$ supersymmetry and must be a calibrated one. The 1-2-3 directions, separated from the others in the above array by the vertical line, are common to all M5-brane worldvolumes. They play an inessential role and may be ignored, as may the transverse tenth direction, so we effectively have three intersecting 2 -branes in $\mathbb{E}^{6}$. The 2-branes in the 6-7 and 8-9 transverse directions may be considered, as before, as 'solitonic' deformations of a test brane in the 4-5 directions. In this case the orthogonal intersection of the three 2-branes can be considered a singular limit of a configuration of a single 2brane 'wrapped' on an elliptic curve in $\mathbb{C}^{3}$, and asymptotic to the three orthogonal 2-planes in the 4-5, 6-7, and 8-9 directions. Each of these planes can be obtained from any of the other two by a discrete $S U(3)$ rotation in $\mathbb{C}^{3}$. Such rotations preserve $1 / 8$ supersymmetry [17]. We can desingularize the intersection, while maintaining $1 / 8$ supersymmetry by allowing a continuous $S U(3)$ rotation from one asymptotic 2-plane to another. We then have a non-singular elliptic curve which, because it preserves $1 / 8$ supersymmetry, must be a calibrated 2 -surface. It must be calibrated by an 
$S U(3)$ Kähler calibration as this is the only candidate with the required properties. This can be verified as before from the constraints imposed on Killing spinors by the intersecting M5-brane configuration.

In any configuration of intersecting branes there will generally be zero modes trapped on the intersection and these will govern its low energy dynamics. In the above example the intersection is 3-dimensional so the zero modes trapped on the intersection yield a $(3+1)$-dimensional quantum field theory. Since the configuration preserves $1 / 8$ of the supersymmetry of the M-theory vacuum, the intersection field theory has a total of four supersymmetries, transforming as a real spinor of $S O(1,3)$. In other words, the low energy intersection dynamics is governed by a $D=4 \mathrm{~N}=1$ SQFT. If the intersection is desingularized so as to describe a single non-singular M5-brane then this SQFT will be determined by the M5-brane's effective action. The SQFT obtained in this way from three orthogonally-intersecting M5-branes is not of particular interest in itself, but any minimal energy M5-brane wrapping a Riemann surface in a flat M-theory vacuum is also calibrated by an $S U(3)$ Kähler calibration, and various SQFTs can be thus obtained. A particularly interesting example in which an M5-brane wraps a Riemann surface in the $S^{1}$-compactified M-theory vacuum was identified in [18] as a theory, now called 'MQCD', with properties similar to that of SQCD.

An example of an associative calibration is provided by four M5-branes intersecting according to the array [5]

$$
\begin{array}{lll|llllllll}
M 5: & 1 & 2 & 3 & 4 & 5 & - & - & - & - & - \\
M 5: & 1 & 2 & 3 & - & - & 6 & 7 & - & - & - \\
M 5: & 1 & 2 & 3 & - & - & - & - & 8 & 9 & - \\
M 5: & 1 & 2 & - & 4 & - & 6 & - & 8 & & -
\end{array}
$$

Ignoring the common directions, and the transverse tenth direction, and interpreting the last three rows as 'solitonic' deformations of a test 3-brane in the 3-4-5 directions, we conclude that we have a calibrated 3 -surface in $\mathbb{E}^{7}$. Since this configuration preserves 1/16 supersymmetry, it must be calibrated by a 3 -form, and the associative 3 -form calibration is the only candidate. We might wish to relate this to a $\mathrm{D}=3$ SQFT, but there is an alternative application. Noting that this array can be obtained from the previous one by the addition of a fourth M5-brane, we retabulate it as

\begin{tabular}{rrrr|rrrrrrr}
$M 5:$ & 1 & 2 & 3 & 4 & 5 & - & - & - & - & - \\
$M 5:$ & 1 & 2 & 3 & - & - & 6 & 7 & - & - & - \\
$M 5:$ & 1 & 2 & 3 & - & - & - & - & 8 & 9 & - \\
& - & - & - & - & - & - & - & - & - & - \\
$M 5:$ & 1 & 2 & - & 4 & - & 6 & - & 8 & - & -
\end{tabular}

If the first three M5-branes are interpreted as supplying a $\mathrm{D}=4 \mathrm{~N}=1 \mathrm{SQFT}$ with coordinates $\left(x^{0}, x^{1}, x^{2}, x^{3}\right)$ then the array suggests that we interpret the fourth M5-brane as a domain wall in this theory. The $1 / 16$ supersymmetry of associative calibrations of an M5-brane translates to $1 / 2$ supersymmetry of the $D=4 N=1$ Minkowski vacuum; in other words, the domain wall is a $1 / 2$ supersymmetric 'BPS' wall. This is a 
simple analogue of Witten's identification of associative calibrations of the M5-brane of MQCD as 1/2 supersymmetric domain walls [18] (see also [19, 20]). It has been known for some time [21, 22] that the WZ model admits $1 / 2$ supersymmetric domain walls for an appropriate superpotential (the solutions having been studied originally as solitons of the dimensionally-reduced $\mathrm{N}=2 \mathrm{D}=2 \mathrm{SQFT}$ [23]). They also appear in $S U(n)$ SQCD with $n \geq 2$ [24] because the low effective action includes a WZ action with a superpotential admitting $n$ isolated critical points and hence $n$ degenerate vacua [25]. Minimal energy configurations that interpolate from one vacuum to another are $1 / 2$ supersymmetric domain walls. It should therefore be no surprise that MQCD admits similar domain walls, although their geometrical nature (and the fact that they are D-branes [18) is remarkable.

It has recently been appreciated that the domain walls of WZ models, and hence of SQCD, may intersect at junctions, the configuration as a whole preserving 1/4 supersymmetry [26, 27]. The junction of two domain walls is 1-dimensional. Let $z$ be a complex coordinate for the plane orthogonal to this direction. The $1 / 4$ supersymmetric domain wall junctions of the WZ model are configurations $\psi(z)$ of a complex scalar field satisfying

$$
\frac{d \psi}{d z}=\overline{W^{\prime}(\psi)}
$$

where the 'superpotential' $W$ is a holomorphic function of $\psi$. The $1 / 2$ supersymmetric domain walls themselves are special solutions of this equation with translational symmetry along one direction in the $z$-plane, but the generic solution is $1 / 4$ supersymmetric. In the case that

$$
W^{\prime}=1-\psi^{3}
$$

corresponding to a quartic superpotential, there are three vacua with $\psi=\left(1, \omega, \omega^{2}\right)$ where $\omega$ is a cube-root of unity. In this case we expect a $\mathbb{Z}_{3}$-symmetric junction, as shown in Fig. 1. Although no exact solution to (26) of this type is known, numerical studies suggest that one exists 28$]$ and the existence of a $\mathbb{Z}_{3}$-invariant minimum energy domain wall junction solution to the second order WZ equations with superpotential (27) has been proved 29]. In addition, an exact solution representing a domain-wall junction has recently been found in a more complicated, but related, model [30]. It seems clear from these results that $1 / 4$ supersymmetric domain wall junctions are generic to $\mathrm{D}=4 \mathrm{~N}=1 \mathrm{SQFTs}$. In particular they are expected in $S U(n) \mathrm{SQFT}$ for $n \geq 3$ 31.

This raises an obvious question. Does MQCD have 1/4 supersymmetric domain wall junctions and, if so, what is their geometrical realization? A suggestion of Gauntlett, which will be explored in a forthcoming article [32], is that domain wall junctions of MQCD are Cayley calibrations of the MQCD M5-brane. This can be motivated by considering the realization of Cayley calibrations as five M5-branes 


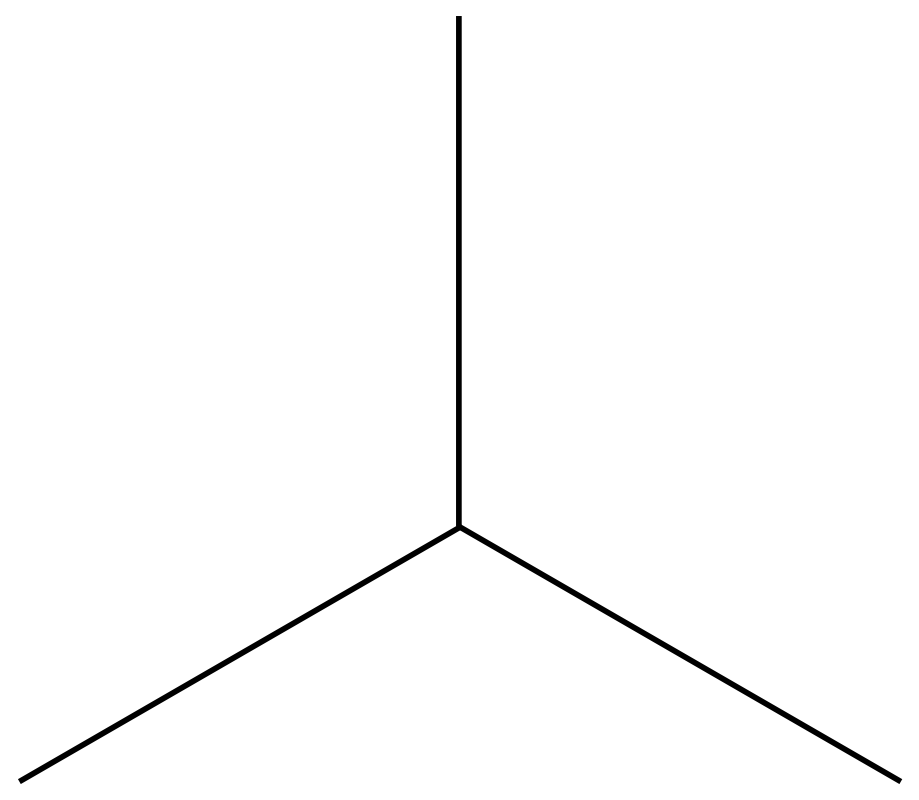

Figure 1: Domain wall junction in the complex z-plane

intersecting orthogonally according to the array [5]

$$
\begin{array}{llll|lllllll}
M 5: & 1 & 2 & 3 & 4 & 5 & - & - & - & - & - \\
M 5: & 1 & 2 & 3 & - & - & 6 & 7 & - & - & - \\
M 5: & 1 & 2 & 3 & - & - & - & - & 8 & 9 & - \\
& - & - & - & - & - & - & - & - & - & - \\
M 5: & 1 & 2 & - & 4 & - & 6 & - & 8 & - & - \\
M 5: & 1 & - & 3 & 4 & - & 6 & - & - & 9 & -
\end{array}
$$

This configuration preserves $1 / 32$ supersymmetry, corresponding to $1 / 4$ supersymmetry of the $\mathrm{D}=4 \mathrm{~N}=1$ vacuum defined by the first three M5-branes. If the last two M5-branes are viewed as excitations about this vacuum then it is apparent from the array that they can be interpreted as intersecting domain walls.

Acknowledgements: I thank Jerome Gauntlett, Gary Gibbons, Jan Gutowski and George Papadopoulos for their collaboration on work reported here, and for helpful discussions.

\section{References}

[1] R. Harvey and H.B. Lawson, Calibrated geometries, Acta. Math. 148 (1982) 47. 
[2] K. Becker, M. Becker, D.R. Morrison, H. Ooguri, Y. Oz and Z. Yin, Supersymmetric cycles in exceptional holonomy manifolds and Calabi-Yau 4-folds, Nucl. Phys. B480 (1996) 225.

[3] S. Stanciu, D-branes in Kazama-Suzuki models, Nucl. Phys. B526 (1998) 295.

[4] G.W. Gibbons and G. Papadopoulos, Calibrations and intersecting branes, Commun. Math. Phys. 202 (1999) 593.

[5] J.P. Gauntlett, N.D. Lambert and P.C. West, Branes and calibrated geometries, Commun. Math. Phys. 202 (1999) 571.

[6] B.S. Acharya, J.M. Figueroa-O'Farrill and B. Spence, Branes at angles and calibrations, JHEP 04:012 (1998); Planes, branes and automorphisms I, JHEP 07 (1998) 004.

[7] B.S. Acharya, J.M. Figueroa-O'Farrill, B. Spence and S. Stanciu, Planes, branes and automorphisms: II. Branes in motion, hep-th/9805176.

[8] J. Gutowski and G. Papadopoulos, adS calibrations, hep-th/9902034.

[9] J. Gutowski, G. Papadopoulos and P.K. Townsend, Supersymmetry and generalized calibrations, Phys. Rev. D60 (1999) 106006.

[10] J.P. Gauntlett, N.D. Lambert and P.C. West, Supersymmetric fivebrane solitons, Adv. Theor. Math. Phys. 3 (1999) 91.

[11] J.P. Gauntlett, Membranes in fivebranes, hep-th/9906162.

[12] J. A. de Azcárraga, J.P. Gauntlett, J.M. Izquierdo and P.K. Townsend, Topological extensions of the supersymmetry algebra for extended objects, Phys. Rev. Lett. 63 (1989) 2443.

[13] E. Bergshoeff, M.J. Duff, C.N. Pope and E. Sezgin, Supersymmetric supermembrane vacua and singletons, Phys. Lett. 199B (1987) 69.

[14] K. Becker, M. Becker and A. Strominger, Fivebranes, membranes and nonperturbative string theory, Nucl. Phys. B456 (1995) 130.

[15] J. Figueroa-O'Farrill, Intersecting brane geometries, hep-th/9806040.

[16] N. Ohta and P.K. Townsend, Supersymmetry of M-branes at angles, Phys. Lett. 418B (1998) 77.

[17] M. Berkooz, M.R. Douglas and R.G. Leigh, Branes intersecting at angles, Nucl. Phys. B480 (1996) 265.

[18] E. Witten, Branes and the dynamics of QCD, Nucl. Phys. B507 (1997) 658. 
[19] V. Kaplunovsky, J. Sonnenschein and S. Yankielowicz, Domain walls in supersymmetric Yang-Mills theories, Nucl. Phys. B552 (1999) 209.

[20] A. Volovich, Domain walls in MQCD and Monge-Ampère equation, Phys. Rev. D59 (1999) 065005.

[21] E.R.C. Abraham and P.K. Townsend, Intersecting extended objects in supersymmetric field theories, Nucl. Phys. B351 (1991) 313.

[22] M. Cvetič, F. Quevedo and S-J Rey, Target space duality and stringy domain walls, Phys. Rev. Lett. 67 (1991) 1836.

[23] P. Fendley, S.D. Mathus, C. Vafa and N.P. Warner, Integrable deformations and scattering matrices for the $N=2$ supersymmetric discrete series, Phys. Lett. 243B (1990) 257.

[24] G. Dvali and M. Shifman, Domain walls and strongly coupled theories, Phys. Lett. 396B (1997) 64; erratum: ibid 407B (1997) 452.

[25] G. Veneziano and S. Yankielowicz, An effective Lagrangian for the pure $N=1$ supersymmetric Yang-Mills theory, Phys. Lett. 113B (1982) 231.

[26] P.K. Townsend and G.W. Gibbons, A Bogomol'nyi equation for intersecting domain walls, Phys. Rev. Lett. 83 (1999) 1727.

[27] S.M. Caroll, S. Hellerman and M. Trodden, Domain wall junctions are 1/4 supersymmetric, hep-th/9905217.

[28] P. Saffin, Tiling with almost-BPS-invaraint domain-wall junctions, Phys. Rev. Lett. 83 (1999) 4249.

[29] L. Bronsard, C. Gui and M. Schatzman, A three-layered minimiser in $\mathbb{R}^{3}$ for a variational problem with a symmetric three-well potential, Commun. Pure Appl. Math. 49 (1996) 677.

[30] H. Oda, K. Ito, M. Naganuma and N. Sakai, An exact solution of BPS domain wall junction, hep-th/9910095.

[31] A. Gorsky and M. Shifman, More on tensorial central charges in $N=1$ supersymmetric gauge theories (BPS wall junctions and strings), hep-th/9909015.

[32] J.P. Gauntlett, G.W. Gibbons and P.K. Townsend, Intersecting domain walls in $M Q C D$, to appear. 